EPJ Web of Conferences 59, 05018 (2013)

DOI: $10.1051 /$ epjconf/20135905018

(C) Owned by the authors, published by EDP Sciences, 2013

\title{
$\alpha$-particle stopping and electron-ion energy relaxation in highly compressed ICF fuel
}

\author{
D.J. Edie ${ }^{1}$, J. Vorberger ${ }^{1}$, S. Rose ${ }^{2}$ and D.O. Gericke ${ }^{1}$ \\ ${ }^{1}$ Centre for Fusion, Space and Astrophysics, Department of Physics, University of Warwick, \\ Coventry CV4 7AL, UK \\ 2 Department of Physics, Imperial College London, London SW7 2BZ, UK
}

\begin{abstract}
We compare calculations for the energy loss of $\alpha$-particles and the electron-ion energy transfer rate in high-density hydrogen plasmas at conditions similar to the compressed main fuel in ICF targets. Different models, which do and do not account for collective effects and electron degeneracy, are considered. It is shown that quantum degeneracy in the cold fuel significantly lowers the energy deposition of the $\alpha$-particles at the high densities of ICF targets. Electron degeneracy is also important for the electronion energy transfer where collective modes can play an important role as well.
\end{abstract}

\section{INTRODUCTION}

The National Ignition Campaign has progressed significantly, with the ultimate goal of controlled laboratory fusion becoming closer. Improvements of the laser drive phase have reduced instabilities [1] and laser-hohlraum coupling has been increased [2] which increases the efficiency of the initial drive phase. In order to achieve efficient energy production the initiation of self-sustaining thermonuclear burn is required. This burning region must spread rapidly to consume a significant fraction of the fuel. Although hotspot ignition is well established, difficulties in reaching the required regime have been encountered and attributed to non-uniformities in pellet compression and fuel mixing [3]. As a result, current investigations into the propagation of an $\alpha$-particle driven burn wave are primarily theoretical.

In this work, the energy deposition of $\alpha$-particles produced by fusion is investigated theoretically. The peak of the energy loss of charged particles is roughly at the thermal speed of the target species and then quickly decreases $[4,5]$. Thus, the dominant contribution to the stopping power stems from target electrons whereas the ion contribution is negligible for most of the stopping process since $3.5 \mathrm{MeV}$ $\alpha$-particles are much faster than most ions in a $\sim 10 \mathrm{keV}$ plasma. Therefore, the energy deposited by the $\alpha$-particles heats the electrons first. Afterwards the ions are heated by electron-ion energy transfer processes. In the central hot spot scenario, the main part of the fuel is at a relatively low temperature and can exhibit significant electron degeneracy. Degeneracy can be quantified by the parameter $n_{e} \Lambda_{e}^{3}$, where $\Lambda_{e}=\left(2 \pi \hbar^{2} / m_{e} k_{B} T_{e}\right)^{1 / 2}$ is the electron thermal wavelength and $n_{e}$ is the electron number density. For plasmas with $n_{e} \Lambda_{e}^{3}>1$, electron degeneracy has significant effects on both the stopping power calculations and the electron-ion relaxation times. Here, we quantify these effects.

\section{APPROACHES FOR THE STOPPING POWER}

For a charged particle travelling through a medium, the rate of change of its kinetic energy is often quantified as the stopping power, $d E / d x$, that is, the kinetic energy lost per unit length. The observed stopping power increases with the density of the medium over a large range of densities, due to the

This is an Open Access article distributed under the terms of the Creative Commons Attribution License 2.0, which permits unrestricted use, distribution, and reproduction in any medium, provided the original work is properly cited. 
increase in the particle collision rate. However, when the stopping medium reaches a density at which electron degeneracy begin to manifest, there is a strong reduction in the rise of the electron contribution with density. As a result, calculations using stopping power models which do not employ the correct physics greatly overestimate the stopping power for high-density systems. Neglecting this quantum effect can lead to a factor of ten or more difference to theories treating it correctly.

A typical example for a stopping power model that neglects quantum degeneracy is the binary collision or T-matrix model of Refs. [6, 7]. Derived from the Boltzmann kinetic equation, the T-matrix model describes the energy loss through binary collision via a statically screened Coulomb potential. Currently, the method used to calculate the scattering cross sections, i.e. the solution of a two-particle Schrödinger equation, prevents the model from including degeneracy effects. Moreover, it does not account for the excitation of collective plasma modes since static screening is considered. Its main advantage is the correct description of strong beam-plasma interactions and the practical fit [8].

Both degeneracy and collective modes can be included when the kinetic approach is based on the quantum Lenard-Balescu kinetic equation [5]. Applied to the stopping power, this approach yields an expression similar to the one derived from dielectric theory (see Refs. [4, 9]) but generalized with respect to quantum diffraction and degeneracy effects in the target medium

$$
\frac{\partial E}{\partial x}=\frac{2 Z_{b}^{2} e^{2}}{\pi v_{b}^{2}} \int_{0}^{\infty} \frac{d k}{k} \int_{\hbar k^{2} / 2 m_{b}-k v_{b}}^{\hbar k^{2} / 2 m_{b}+k v_{b}} d \omega\left(\omega-\frac{\hbar k^{2}}{2 m_{b}}\right) n_{B}(\omega) \operatorname{Im} \varepsilon^{-1}(k, \omega) .
$$

Here, $Z_{b}$ refers to the charge state of the beam particle, $v_{b}$ is its velocity, and $m_{b}$ is its mass. $\varepsilon$ is the dielectric function of the taget medium which is calculated using the random phase approximation (RPA). $n_{B}$ denotes the Bose function describing the collective excitations.

The RPA description of the stopping power (1) is strictly valid for weak beam-particle interactions only but fully accounts for collective effects and arbitrary degeneracy. In this model, the energy loss is governed by interactions of the beam particles with the mean field and the collective modes of the plasma. For ICF conditions, the use of improved models for the dielectric function, i.e., the Mermin approach or local field corrections, changes the results only marginally.

The disadvantage of the approach (1) is its computational cost. Thus, it is often convenient to employ a reduced model which interpolates between limiting cases. One may summarize a collisional model (see Ref. [10] for an extended discussion) for the stopping power by

$$
\frac{d E}{d x}=-\frac{4 \pi Z_{b}^{2} e^{4}}{m_{e} v_{b}^{2}} n_{e} \ln \Lambda\left[\operatorname{erf}\left(x_{0}\right)-\left(1+\frac{m_{e}}{m_{b}}\right) \frac{2}{\sqrt{\pi}} x_{0} \exp \left(-x_{0}^{2}\right)\right],
$$

where

$$
\begin{aligned}
x_{0} & =\left(\frac{m_{e} \epsilon_{b}}{m_{b} k_{B} T_{e}}\right)^{1 / 2}\left[\frac{\sqrt{\pi}}{2 I_{1 / 2}(\eta)\{1+\exp (-\eta)\}}\right]^{1 / 3}, \\
\Lambda & =\frac{2 m_{e} v_{e}^{2}}{\hbar \omega_{p e}} \frac{0.321+0.259 x_{0}^{2}+0.0707 x_{0}^{4}+0.05 x_{0}^{6}}{1+0.130 x_{0}^{2}+0.05 x_{0}^{4}} .
\end{aligned}
$$

Here, $\epsilon_{b}=m_{b} v_{b}^{2} / 2$ and $\eta=\mu / k_{B} T$, where $\mu$ is the chemical potential. $\omega_{p e}=\sqrt{4 \pi n_{e} e^{2} / m_{e}}$ denotes the electron plasma frequency and $I_{1 / 2}$ is the Fermi integral to order $1 / 2$. We refer to this expression as the 'Thomas-Fermi model' as it originates from attempts using a local density calculated by a hightemperature Thomas-Fermi model. Here, it is however employed for a fixed, average electron density.

Fig. 1 shows a comparison of the three stopping power models discussed for cases at varying levels of electron degeneracy in the target. In cases of highest and lowest degeneracy, the Thomas-Fermi model approaches the Lenard-Balescu results. This behaviour is also found for higher degeneracy or fully 

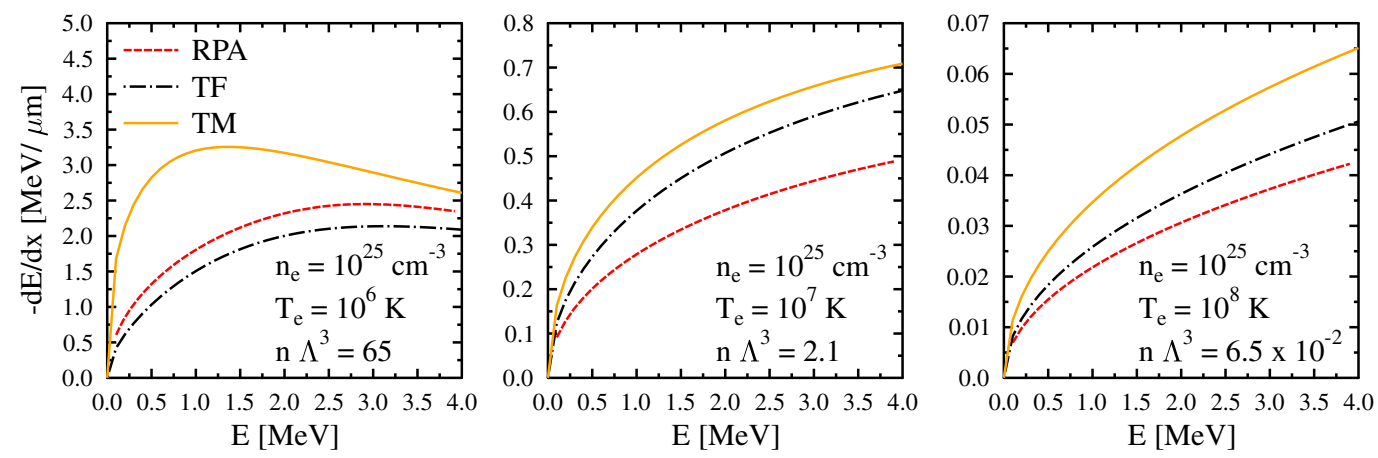

Figure 1. Comparison of stopping power calculated by different models for a range of plasma parameters. Cases shown include weak, intermediate and strong degeneracy. The stopping models used are T-matrix (TM), the LenardBalescu dielectric (RPA) and Thomas-Fermi model (TF); see text.

classical systems. In the highest degeneracy case shown, the T-matrix model significantly deviates from the other stopping models. These differences become more significant as the degeneracy of the stopping medium is further increased. For the nondegenerate system, all models show similar behaviour but the Thomas-Fermi and Lenard-Balescu models return lower values of stopping power for these low beam energies.

\section{RATES OF ELECTRON-ION ENERGY TRANSFER}

In typical ICF cases, the energy deposition of the $\alpha$-particles heats the electrons and not the ions. In this situation, ions gain energy via subsequent electron-ion energy transfer processes. Considering binary collisions only, the energy transfer can be described by a T-matrix approach similar to the one for the stopping power [11]

$$
\frac{\partial}{\partial t} E_{i}=Z_{e i}(t)=\int \frac{d \tilde{\mathbf{p}}}{2 \pi^{2} \hbar^{3}} \frac{f_{i}(\tilde{\mathbf{p}})}{n_{i}} \frac{n_{e} \Lambda_{e}^{3}}{(2 \pi)^{2} \hbar^{3} m_{e}} \frac{k_{B} T_{e}(t)}{\tilde{p}} \int_{0}^{\infty} d k k^{3} Q_{e i}^{T}(k)\left[k_{-} e^{-\frac{m_{e} v_{-}^{2}}{2 k_{B} T_{e}(t)}}-k_{+} e^{-\frac{m_{e} v_{+}^{2}}{2 k_{B} T_{e}(t)}}\right] .
$$

Here, $Q_{e i}^{T}$ is the electron-ion momentum transport cross-section. Furthermore, we have introduced the abbreviations $k_{ \pm}=k \pm \tilde{p}+m_{i} k_{B} T_{e} / k$ and $v_{ \pm}=k / m_{e} \pm \tilde{p} / m_{i}$. Eq. (5) describes temperature relaxation through binary collisions only but is also valid for strong electron-ion coupling. Thus, it generalizes the seminal Landau-Spitzer approach [11].

To account for the collective behaviour of a plasma, the electron-ion energy transfer process must be described via an approach that considers coupled collective modes and energy transfer through mode coupling [12]. For weakly coupled plasmas, this approach can be based again on the Lenard-Balescu kinetic equations [13] or more generally on a Green's function approach [12, 14] yielding

$$
Z_{e i}(t)=-4 \mathcal{V} \hbar \int \frac{d^{3} \mathbf{q}}{(2 \pi \hbar)^{3}} \int_{0}^{\infty} \frac{d \omega}{2 \pi} \omega \Delta N_{B}(\omega) \frac{\operatorname{Im} \varepsilon_{e}^{R}(\mathbf{q} ; \omega, t) \operatorname{Im} \varepsilon_{i}^{R}(\mathbf{q} ; \omega, t)}{\left|\varepsilon^{R}(\mathbf{q} ; \omega, t)\right|^{2}},
$$

with the dielectric function of the fully coupled system given as

$$
\varepsilon^{R}(\mathbf{q} ; \omega, t)=1-\sum_{a} V_{a a}(\mathbf{q}) \Pi_{a}^{R}(\mathbf{q} ; \omega, t) .
$$

$\Delta N_{B}(\omega)=n_{B}\left(\omega / T_{e}\right)-n_{B}\left(\omega / T_{i}\right)$ is the difference of Bose functions with electron and ion temperature, respectively, $V_{a a}$ is the Coulomb potential, $\Pi_{a}^{R}$ is the polarisation function and $\mathcal{V}$ represents the volume 

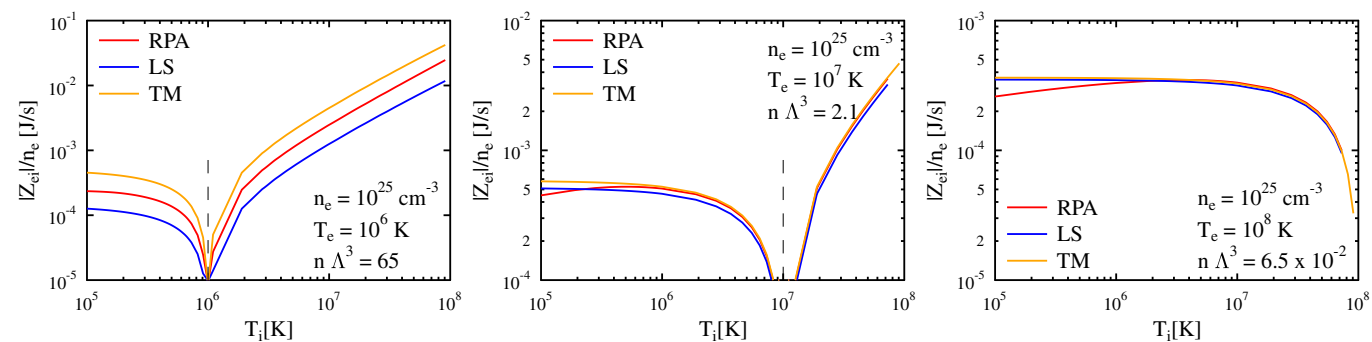

Figure 2. Electron-ion energy transfer rates for the same plasma parameters as in Fig. 1. Theories used are the coupled mode description (7) with a dielectric function in RPA, the Landau-Spitzer formula (LS) $[15,16]$ and the full binary collision approach (TM) [11].

of the system. Electron-ion energy transfer calculations based on this expression account for both degeneracy and collective effects. As with the dielectric model of stopping power, it is computationally intensive to solve the double integral in Eq. (6).

Figure 2 shows results for the electron-ion energy transfer rates in a hydrogen plasma for similar conditions as used in Fig. 1. For the nondegenerate case, all energy transfer rates are identical, except for large temperature differences between electrons and ions where the formula of Eq. (6) predicts a lowered energy transfer of up to a factor of two due to mode coupling effects. The same agreement holds for the case of intermediate degeneracy between T-matrix and coupled mode energy transfer rates. However, the LS rate already predicts a consistently lower energy transfer, which is a precursor for a break-down of the LS description of collisions (see Ref. [11] for a detailed discussion). For the example with high degeneracy, predictions for the energy transfer between the three models presented here differ by a factor of four. Due to the physics included, we judge that the most reliable theory is the coupled mode description Eq. (6) which is underestimated by the LS formula due to the break-down of the collision model and overestimated by the T-matrix model due to the neglection of degeneracy.

\section{CONCLUDING REMARKS}

This investigation aims to create the basis for $\alpha$-particle heating in ICF pellets. The comparisons show that electron degeneracy must be incorporated in all energy transfer processes: $\alpha$-particles generated in burning core heat a larger spatial region than predicted without degeneracy. Electron degeneracy also modifies the electron-ion energy transfer. When applied to hot spot scenarios, the combined effects of modified $\alpha$-particle stopping and the heat transfer to the ions give a substantially increased time before the cold, dense fuel reaches ignition temperatures. Accordingly, one would predict a reduction in the propagation velocity of an $\alpha$-particle driven burn wave and, thus, less efficient burn.

\section{References}

[1] P. Michel et al. Phys. Rev. E 83, 046409 (2011)

[2] S. H. Glenzer et al. Phys. Rev. Lett. 106, 085004 (2011)

[3] B. A. Hammel et al. Phys. Plasmas 18, 056310 (2011)

[4] N. R. Arista and W. Brandt, Phys. Rev. A 23, 1898 (1981)

[5] W.-D. Kraeft \& B. Strege, Physica A 149, 313 (1988)

[6] D. O. Gericke, M. Schlanges and W.-D. Kraeft, Phys. Lett. A 222, 241 (1996)

[7] D. O. Gericke \& M. Schlanges, Phys. Rev. E 60, 904 (1999)

[8] D. O. Gericke, Laser and Particle Beams 20, 471 (2002)

[9] C. Gouedard and C. Deutsch, J. Math. Phys. 19, 32 (1978) 


\section{IFSA 2011}

[10] S. Skupsky, Phys. Rev. A 16, 727 (1977)

[11] D. O. Gericke, M. S. Murillo and M. Schlanges, Phys. Rev. E 65, 036418 (2002)

[12] M. W. C. Dharma-wardana and F. Perrot, Phys. Rev. E 58, 3705 (1998)

[13] J. Vorberger and D. O. Gericke, Phys. Plasmas 16, 082702 (2009)

[14] J. Vorberger, D. O. Gericke, Th. Bornath and M. Schlanges, Phys. Rev. E 81, 046404 (2010)

[15] L. D. Landau, Phys. Z. Sowjetunion 10, 154 (1936)

[16] L. Spitzer, Physics of Fully Ionized Gases (Interscience, New York, 1962) 